\title{
Cerebral metastasis with 18F-FDG uptake deficiency from non small-cell lung cancer
}

\author{
Wang $\mathrm{X}^{* 1}$, Jin $\mathrm{H}^{2}$, Wang $\mathrm{F}^{3}$, Liu $\mathrm{J}^{\# 4}$ and $\operatorname{Li} \mathrm{Y}^{\# 5}$ \\ ${ }^{1}$ Department of PET/CT, Lanzhou University Second Hospital, Lanzhou, Gansu province, China \\ ${ }^{2}$ Department of Radiology, Washington University School of Medicine, St. Louis, MO, USA \\ ${ }^{3}$ Department of Pathology, Lanzhou University Second Hospital, Lanzhou, Lanzhou, Gansu province, China \\ ${ }^{4}$ Department of PET/CT, Lanzhou University Second Hospital, Lanzhou, Gansu province, China \\ ${ }^{5}$ Department of General Surgery, Lanzhou University Second Hospital, Lanzhou,Gansu province, China \\ \#Equally contributed
}

\begin{abstract}
Lung cancers form a leading cause of death worldwide. The prognosis in lung cancer is greatly worsened by the presence of metastases, the brain is an extremely common metastatic site. Non small-cell lung cancer (NSCLC) was more frequently associated with hypermetabolic metastatic brain lesions than small-cell lung cancer (SCLC). ${ }^{18} \mathrm{~F}$-FDG PET/CT is increasingly used in detecting and staging of lung cancer as single "one stop shop" method. However FDG uptake deficiency from NSCLC has not well addressed. Here we presented a particular case of ${ }^{18} \mathrm{~F}$-FDG PET/CT for cerebral metastasis with ${ }^{18} \mathrm{~F}$-FDG uptake deficiency from NSCLC.
\end{abstract}

\section{Case presentation}

A 60-year-old Chinese man was admitted to Hospital with the history of intermittent convulsion of left upper limb with numbness of left lower limb for more than half a month, without headache, dizziness, nausea, vomiting and other symptoms. Brain magnetic resonance imaging (MRI) revealed an occupying lesion in right frontal lobe (Figure $1 \mathrm{~A}-\mathrm{C}$ ), then brain metastasis and gliomas were considered. A chest X-ray was taken, and no abnormality was detected. Tumor markers were slightly higher and results were summarized. Subsequently, he was referred for an FDG PET/CT imaging to evaluate the whole-body, the images revealed a low-density lesion in right frontal lobe with distinct borderline, and FDG uptake was deficient completely, less than that of white matter (Figure 2). Meanwhile, a solitary pulmonary nodule was detected in upper lobe of the right lung with higher FDG uptake (standardized uptake values (SUVmax: 2.1) (Figure 3), without any other abnormality in other organs.

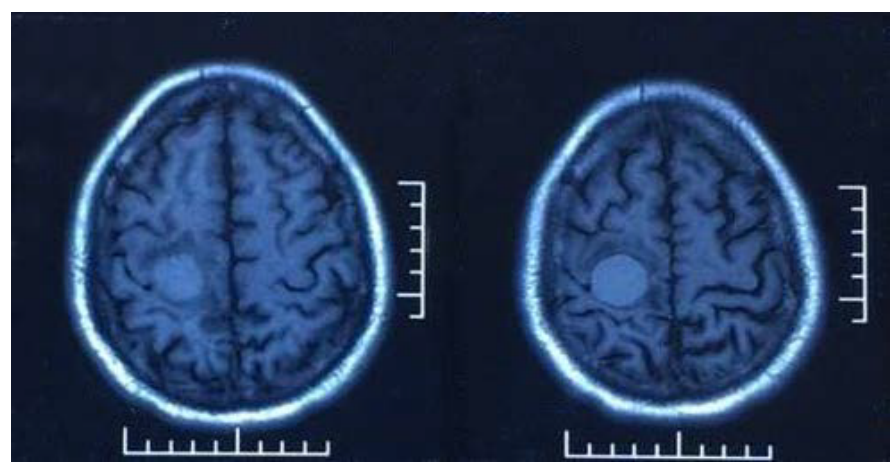

Figure 1A. Brain MRI reveals a mass lesion in right frontal lobe, it appears hyperintense on both $\mathrm{T} 1$-weighted and $\mathrm{T} 2$-weighted images.

\section{Discussion}

Lung cancers form a leading cause of death worldwide, of which more than $80 \%$ show non-small cell histology [1]. Unfortunately, most lung cancers present at a clinically advanced stage $[2,3]$. PET/CT is now an important cancer imaging tool, both for diagnosis and staging, as well as offering prognostic information based on response.

Because of the high FDG uptake in gray matter, the contrast of the uptake from the tumor versus normal brain tissue decreases, leading to low sensitivity. It is accepted that the correlation of grades with ${ }^{18} \mathrm{~F}-\mathrm{FDG}$ showed high-grade brain tumor demonstrated hypermetabolism with high FDG uptake, while low-grade brain tumors revealed low levels of glucose metabolism with less than, equal to, or slightly higher than that of white matter. Cerebral metastases mainly occur in the supratentorial

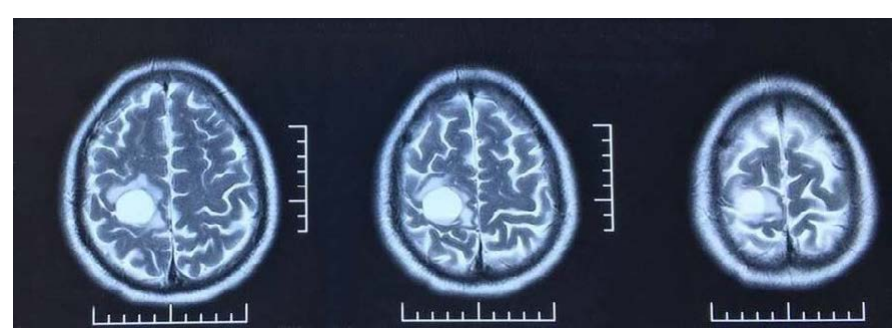

Figure 1B. T2-weighted images and contrast-enhanced MRI.

Correspondence to: Xiaohui Wang, Lanzhou University Second Hospital, PET/ CT Department, Lanzhou, Gansu province, China, E-mail: 1394552545@qq.com Key words: non-small-cell lung cancer, brain metastasis, 18F-FDG PET/CT

Received: January 29, 2017; Accepted: February 21, 2017; Published: February 24, 2017 


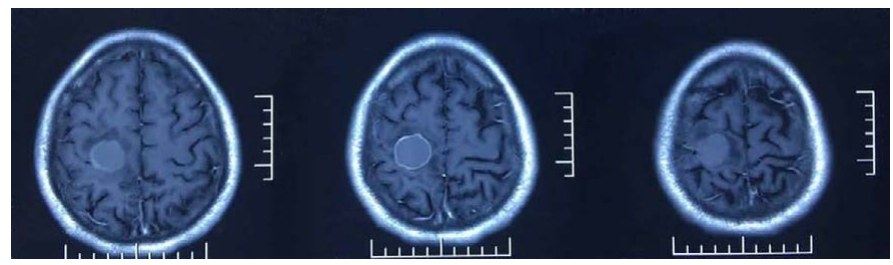

Figure 1C. Showing the lesion is ring-enhanced, peritumoral edema is slight, and without midline shift.
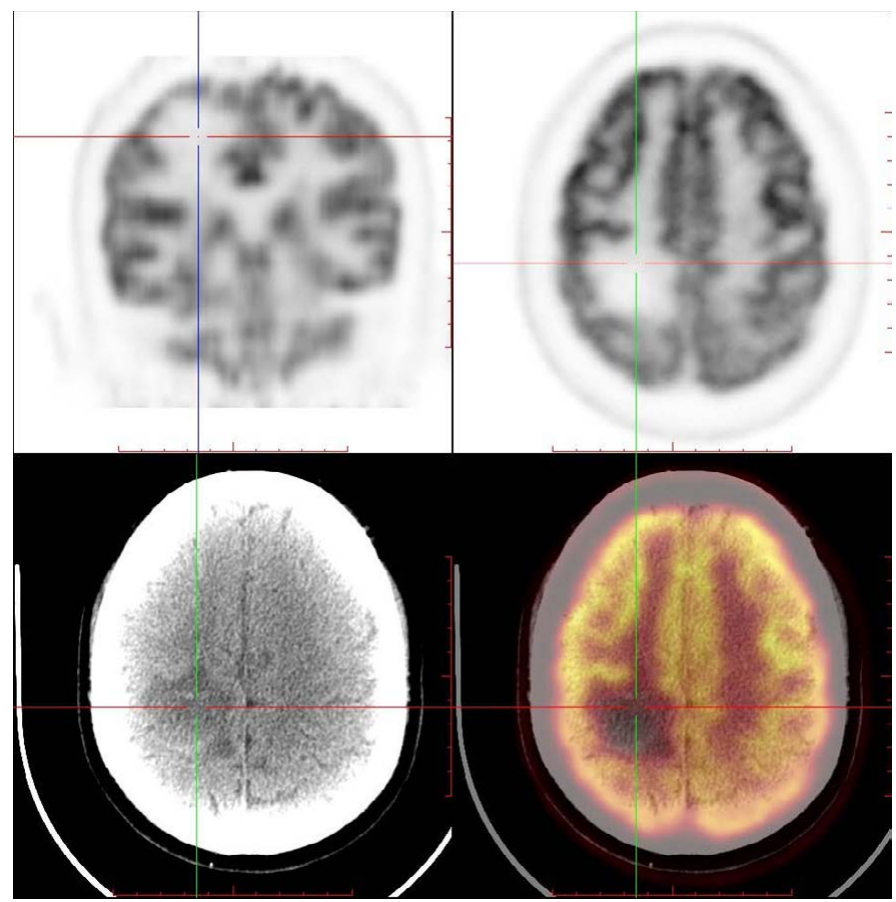

Figure 2. FDG PET/CT images demonstrates a focal FDG uptake deficiency in right frontal lobe, and non-contrast CT images also show a hypodense lesion at the region accordingly (35 mm x $34 \mathrm{~mm} \times 18 \mathrm{~mm}$ ).

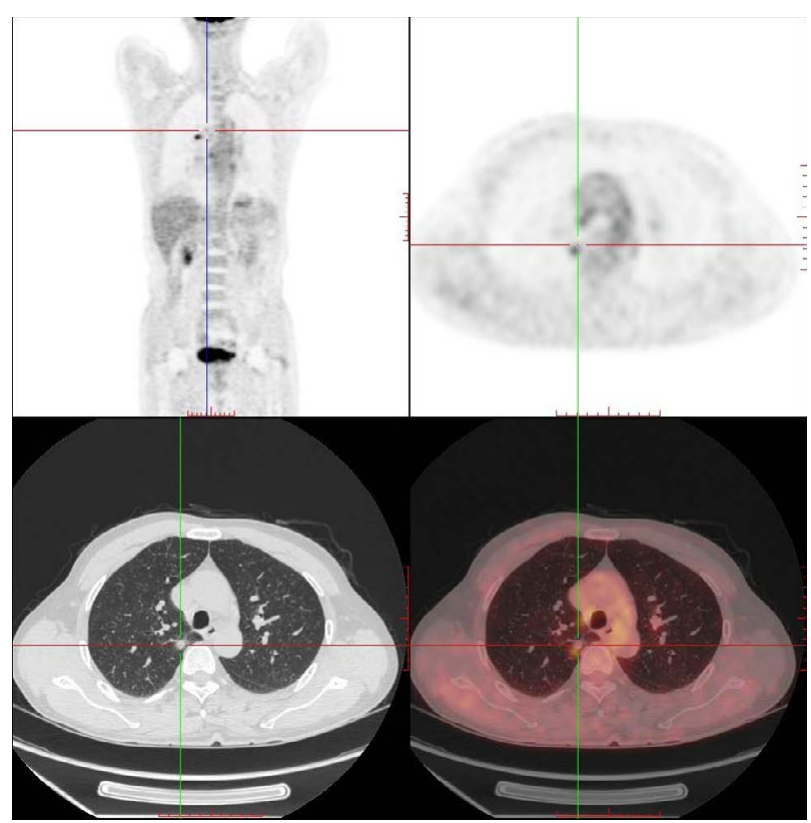

Figure 3. FDG PET/CT images show a solitary pulmonary nodule $(9.8 \mathrm{~mm} \times 12 \mathrm{~mm} \times 11$ $\mathrm{mm}$ ) in upper lobe of the right lung with focal increased hypermetabolism (SUVmax:2.1), and its edge is vague.
$(80 \%)$ and characterized by significant cerebral edema. As for this case, the edema is slightly both from MR and CT brain scan, and the FDG uptake is deficient completely, and the patient was wrongly regarded as low-grade gliomas initially, and lung cancer in right upper lobe was highly suspicious. Then surgical resection was undertaken to remove the lesion in the right frontal lobe, and the pathology diagnosis was metastatic adenocarcinoma (Figure 4 A-D). The case was finally conformed cerebral metastasis from NSCLC, and the slightly higher CEA also aided the diagnosis.

The management of patients with single brain metastasis (BM) from NSCLC is controversial [4]. Surgical resection with adjuvant irradiation and stereotactic radiosurgery (SRS) are performed in the treatment of such lesions, Emilie Bougie et al concluded that patients with single BM undergoing surgical resection had a survival advantage [5].

A larger scale study declared that NSCLC was more commonly

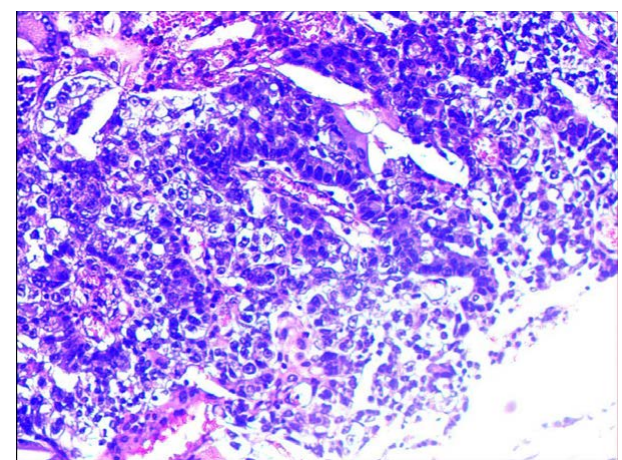

Figure 4A. HE staining: Microscopic examination of brain tumor lesion shows tumor cells with tubular formation, nuclear enlargement, nuclear fission, which suggest metastatic brain tumor.

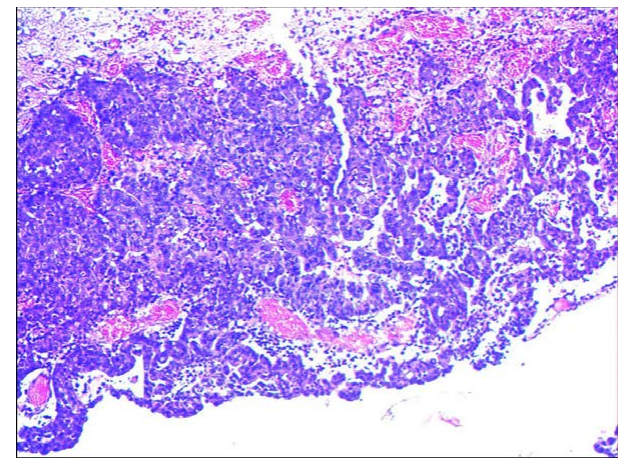

Figure 4B. HE staining: Microscopic examination of brain tumor lesion shows tumor cells with tubular formation, nuclear enlargement, nuclear fission, which suggest metastatic brain tumor.

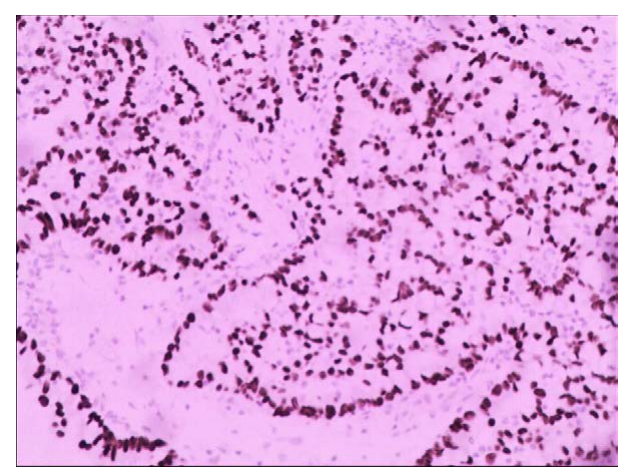

Figure 4C. Immunohistochemical staining: TTF-1(+), CK8-18(+). 


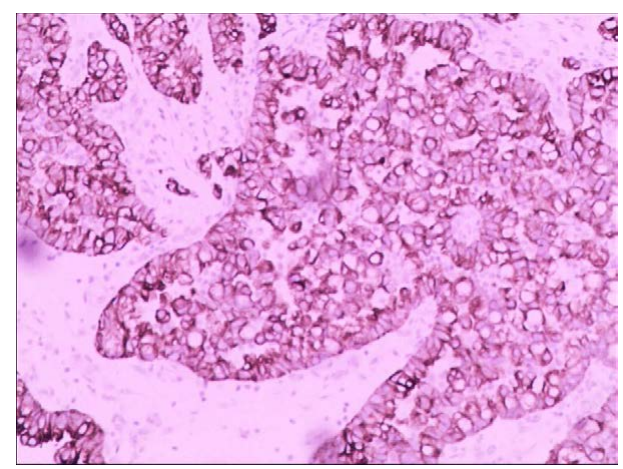

Figure 4D. Which suggest the brain metastasis originates from lung adenocarcinoma.

associated with hypermetabolic rather than hypometabolic metastatic brain lesion. The PET findings of brain lesions were affected not only by the size of lesion but also by its biological characteristics [6]. One or several foci of increased ${ }^{18} \mathrm{~F}-\mathrm{FDG}$ is highly suspicious of metastases. However, the lack of ${ }^{18} \mathrm{~F}-\mathrm{FDG}$ uptake does not rule out metastasis, this case illustrated typically. The reason for the ${ }^{18} \mathrm{~F}-\mathrm{FDG}$ uptake deficiency was chronic hemorrhage, which can be concluded from MR T1WI signal (brighter than brain tissue).

This case also confirmed that chest X-ray is not far enough to rule out pulmonary mass and tumor markers is a useful indicator for lung cancer diagnosis, even it is slightly higher.

\section{Highlights}

1. PET/CT is considered as single "one stop shop" method for
2. 18F-FDG uptake deficiency from Non small-cell lung cancer has not well addressed.

3. A case of cerebral metastasis with 18 F-FDG uptake deficiency was presented.

\section{Conclusion}

For patients with cerebral metastasis, whole-body ${ }^{18} \mathrm{~F}-\mathrm{FDG}$ PET/ CT scans were useful in finding the primary cancer in the body. In ${ }^{18} \mathrm{~F}$-FDG images, one or several foci of increased ${ }^{18} \mathrm{~F}$-FDG activity is highly suspicious of metastases. However, the deficiency of ${ }^{18} \mathrm{~F}-\mathrm{FDG}$ uptake does not rule out metastasis, hemorrhage is one of its causes.

\section{References}

1. Na II, Lee TH, Choe DH (2008) A diagnostic model to detect silent brain metastases in patients with non-small cell lung cancer. European Journal of Cancer 44: 2411-2417.

2. Ebben JD, You M (2016) Brain metastasis in lung cancer: Building a molecular and systems-level understanding to improve outcomes. The International Journal of Biochemistry \& Cell Biology 78: 288-296.

3. DuellT, Kappler S, Knöferl B (2015) Prevalence and risk factors of brain metastases in patients with newly diagnosed advanced non-small-cell lung cancer. Cancer Treatment Communications 4: 106-112.

4. Pollock BE (2015) To Remove or Not to Remove, that Is the Question? World Neurosurgery 84: 2-3.

5. Bougie E, CôtéML, Mathieu D (2015) Comparison Between Surgical Resection an Stereotactic Radiosurgery in Patients with a Single Brain Metastasis from Non-Smal Cell Lung Cancer. World Neurosurgery 83: 900-906.

6. Lee HY, Chung JK, JeongJM (2008) Comparison of FDG-PET findings of brain metastasis from non-small-cell lung cancer and small-cell lung cancer. Annals of nuclear medicine 22: 281-286. cancer.

Copyright: $@$ 2017 Wang X. This is an open-access article distributed under the terms of the Creative Commons Attribution License, which permits unrestricted use, distribution, and reproduction in any medium, provided the original author and source are credited. 